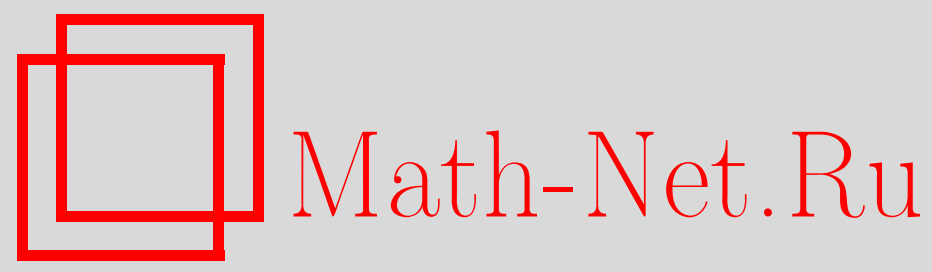

В. В. Осокин, О сложности расшифровки разбиения булева куба на подкубы, Дискрет. матем., 2008, том 20, выпуск $2,46-62$

DOI: https://doi.org/10.4213/dm1003

Использование Общероссийского математического портала Math-Net.Ru подразумевает, что вы прочитали и согласны с пользовательским соглашением http://www . mathnet.ru/rus/agreement

Параметры загрузки:

IP : 54.80 .97 .219

26 апреля 2023 г., 10:06:20 


\title{
О сложности расшифровки разбиения булева куба на подкубы
}

\author{
() 2008 г. $\quad$ В. В. Осокин
}

\begin{abstract}
Рассматривается известна задача расшифровки функций алгебры логики, то есть задача восстановления значений функции на всех наборах $n$-мерного булева куба по известным значениям на некоторых из этих наборов. В общем случае для определения функции нужно знать ее значения на всех $2^{n}$ наборах. Но если функция принадлежит некоторому более узкому классу, чем класс всех функций алгебры логики от $n$ переменных, то для ее определения могут потребоваться лишь значения на некотором подмножестве $n$-мерного булева куба. В данной работе рассматриваются классы функций, производящих разбиение $n$-мерного булева куба на подкубы. Получены асимптотические оценки сложности расшифровки функций из таких классов.
\end{abstract}

\section{1. Введение}

В последние годы все более актуальной становится проблема организации эффективного доступа к информации, доступной через такие источники как Интернет. Широко известные и распространенные модели информационного поиска документов, такие, как булева, векторная и вероятностная [1], уже не удовлетворяют в полной мере требованиям, предъявляемым к качеству поиска. Вызвано это, в частности, тем, что указанные модели не используют связи (так называемые тематические связи) между словами и документами, которые можно получить за счет анализа имеющегося корпуса документов. Чтобы преодолеть этот недостаток активно разрабатываются новые методы информационного поиска документов (в основном, на базе упомянутых выше моделей). К таким методам можно, например, отнести LSI [2] и PLSI [3]. Эти методы позволяют разбить множество документов на подмножества, документы в которых тематически схожи. При этом анализ релевантности документа запросу пользователя производится уже не сравнением слов, встречающихся в документе и запросе, а сравнением тем, в которых лежат документ и запрос. Это позволяет расширить и улучшить результаты поиска, в которых при таком подходе могут появиться даже релевантные документы, тематически схожие с запросом, но не содержащие слов из запроса.

То, насколько хорош тот или иной метод тематического поиска документов, можно, например, оценивать по точности, с которой он разбивает корпус документов по темам. При этом такая оценка производится в большинстве случаев чисто экспериментально, так как про реальное разбиение корпуса по темам обычно ничего не известно. Широко распространены тестовые корпуса для подобного рода экспериментов [1], подготавливаемые вручную. Понятно, что такие корпуса не могут служить основой для точных теоретических оценок. С другой стороны, для целого ряда задач информационного поиска подобные 
оценки имеются [4], что стимулирует дальнейшие теоретические исследования описанной задачи.

Существуют работы, в которых понятия корпуса документов и темы вводятся математически (например, [5]) с использованием вероятностных распределений. Но результаты в данном направлении далеки от окончательных.

В настоящей работе мы не фиксируем разбиение корпуса документов по темам, но в то же время накладываем на темы некоторые (достаточно существенные) ограничения, которые позволяют точно и однозначно определять разбиение корпуса документов по темам. Это позволяет построить алгоритм, производящий разбиение корпуса документов по темам, и показать его асимптотическую неулучшаемость.

Будем считать, что имеется упорядоченное множество из $n$ слов. Полагаем, что документ - это булев вектор длины $n$, на $i$-м месте которого стоит 1 , если $i$-е слово есть в документе, и 0 в противном случае. Таким образом, документы можно понимать как точки $n$-мерного булева куба. Полагаем также, что имеется множество тем и что каждый из рассматриваемых $2^{n}$ документов принадлежит некоторой (одной) теме из этого множества. Таким образом, существует некоторая функция, сопоставляющая каждому документу номер темы, в которой он лежит, назовем эту функцию темоопределяющей (ТОФ). Предполагаем, что каждая тема задается некоторой элементарной конъюнкцией на множестве

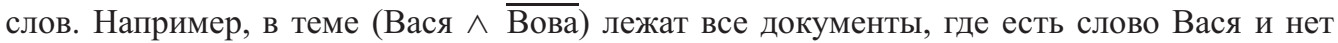
слова Вова, и только они. Сама ТОФ неизвестна, и, что то же самое, неизвестны слова, образующие темы (тематические слова). Но при этом полагаются известными структура и количество тем, то есть мы знаем, что есть тема (слово $1 \wedge \overline{\text { слово }_{2}}$, но не знаем, что слово $_{1}$ - это Вася, а слово 2 - это Вова. Требуется определить тематические слова, и следовательно, полностью определить ТОФ.

В данной работе мы сведем эту задачу к задаче расшифровки функций из заданного класса. В нашем случае это класс функций, задающих одинаковые по структуре разбиения куба. Будем, как и в [6], предполагать, что есть некоторый оператор $\mathscr{A}_{f}$, действующий из множества документов во множество тем, который по заданному документу выдает значение ТОФ $f$ на этом документе (то есть тему, в которой этот документ лежит). Любой алгоритм, решающий поставленную задачу, может запрашивать значения оператора $A_{f}$ на любых наборах $n$-мерного булева куба. Очевидно, что запросив значения $A_{f}$ на всех $2^{n}$ наборах, мы полностью определим ТОФ $f$ и, значит, решим задачу. В данной работе строится алгоритм, решающий задачу, у которого число обращений к оператору $A_{f}$ асимптотически равно $k \log _{2} n$, где $k$ - число существенных переменных функции $f$. Кроме того, получена нижняя оценка сложности определения ТОФ, доказывающая асимптотическую оптимальность данного алгоритма.

Автор благодарен Э. Э. Гасанову за постановку задачи и помощь в работе.

\section{2. Постановка задачи и формулировка результатов}

Напомним, что $k$-мерным булевым кубом называется множество наборов

$$
B^{k}=\left\{\left(\alpha_{1}, \ldots, \alpha_{k}\right): \alpha_{i} \in\{0,1\}, i=1, \ldots, k\right\} .
$$

Подкубом $k$-мерного булева куба $B^{k}$ называется каждое множество вида

$$
B_{\sigma_{1}, \ldots, \sigma_{s}}^{k, i_{1}, \ldots, i_{s}}=\left\{\left(\alpha_{1}, \ldots, \alpha_{k}\right) \in B^{k}: \alpha_{i_{1}}=\sigma_{1}, \ldots, \alpha_{i_{s}}=\sigma_{s}\right\}
$$


Множество $\left\{i_{1}, \ldots, i_{s}\right\}$ называется направлением, число $s-$ рангом, а число $k-s-$ размерностью подкуба $B_{\sigma_{1}, \ldots, \sigma_{s}}^{k, i_{1}, \ldots, i_{s}}$.

Через $x_{i}^{\sigma}, i=1, \ldots, k, \sigma \in\{0,1\}$, обозначим булеву функцию, принимающую значения 1 в точности на всех таких наборах $\left(\alpha_{1}, \ldots, \alpha_{k}\right) \in B^{k}$, что $\alpha_{i}=\sigma$. Далее будем также использовать обозначения $x_{i}^{1}=x_{i}, x_{i}^{0}=\bar{x}_{i}$. Тогда понятно, что элементарная конъюнкция $x_{i_{1}}^{\sigma_{1}} \ldots x_{i_{s}}^{\sigma_{s}}$ принимает значение 1 в точности на подкубе $B_{\sigma_{1}, \ldots, \sigma_{s}}^{k, i_{1}, \ldots, i_{s}}$. Таким образом, каждому подкубу поставлена в соответствие некоторая элементарная конъюнкция.

Тематическим разбиением $R$ булева $k$-мерного куба назовем произвольное упорядоченное множество подкубов этого куба такое, что

(a) пересечение любых двух элементов $R$ пусто;

(б) объединение всех элементов $R$ дает $B^{k}$; для любой переменной $x_{j}, j=1, \ldots, k$, существует такой элемент множества $R$, что переменная $x_{j}$ входит в соответствующую этому элементу элементарную конъюнкцию.

Последнее условие состоит в том, что все $k$ переменных существенны в разбиении.

Элементы тематического разбиения $R$ будем называть темами; $k$-мерный булев куб с фиксированным тематическим разбиением $R$ назовем тематическим $R$-кубом.

Тематический $R$-куб можно описывать с помощью ДНФ, являющейся дизъюнкцией элементарных конъюнкций, соответствующих темам из $R$. Понятно, что эта ДНФ реализует тождественную единицу. В дальнейшем, когда это удобно, будем подразумевать под тематическим разбиением куба именно эту ДНФ. Будем также предполагать, что порядковый номер конъюнкции в ДНФ задает номер темы, и для любой темы $t \in R$ через $\mathcal{N}(t)$ обозначать номер этой темы.

Рассмотрим в качестве примера тематическое разбиение $R_{0}$

$$
\underbrace{x_{2} x_{3}}_{1} \vee \underbrace{x_{1} \bar{x}_{3}}_{2} \vee \underbrace{\bar{x}_{1} \bar{x}_{2}}_{3} \vee \underbrace{x_{1} \bar{x}_{2} x_{3}}_{4} \vee \underbrace{\bar{x}_{1} x_{2} \bar{x}_{3}}_{5}
$$

куба $B^{3}$. В данном случае имеется 5 тем. Соответствующие подкубы $B^{3}$ выделены жирным на рис. 1 .

Пусть $k, n$ - натуральные числа, $k \leqslant n, R$ - тематическое разбиение $k$-мерного куба, $\left\{y_{1}, \ldots, y_{n}\right\}-$ множество булевых переменных.

Функцию $f\left(y_{1}, \ldots, y_{n}\right): B^{n} \rightarrow\{1,2, \ldots,|R|\}$ будем называть темоопределяющей (ТОФ) над разбиением $R$ булева $k$-мерного куба, если существует упорядоченное множество индексов $q_{1}, \ldots, q_{k}$ такое, что

(a) $q_{j} \in\{1,2, \ldots, n\}$ и $q_{j_{1}} \neq q_{j_{2}}$, если $j_{1} \neq j_{2}, j, j_{1}, j_{2} \in\{1, \ldots, k\}$;

(б) для произвольной $j$-й темы, $j \in\{1,2, \ldots,|R|\}, x_{i_{1}}^{\sigma_{1}} \ldots x_{i_{t_{j}}}^{\sigma_{t_{j}}}$ разбиения $R$ из того, что $y_{q_{i_{1}}}=\sigma_{1}, \ldots, y_{q_{i_{j}}}=\sigma_{t_{j}}$ следует, что $f\left(y_{1}, \ldots, y_{n}\right)=j$.

При этом множество индексов $\left\{q_{1}, \ldots, q_{k}\right\}$ будем называть основанием темоопределяющей функции $f$, а переменные $y_{q_{1}}, \ldots, y_{q_{k}}$ - тематическими переменными функции $f$. Положим $y_{q_{1}}=\Psi\left(x_{1}\right), \ldots, y_{q_{k}}=\Psi\left(x_{k}\right)$.

Приведем пример ТОФ для разбиения $R_{0}$, изображенного на рис. 1 . Положим $n=30$. 


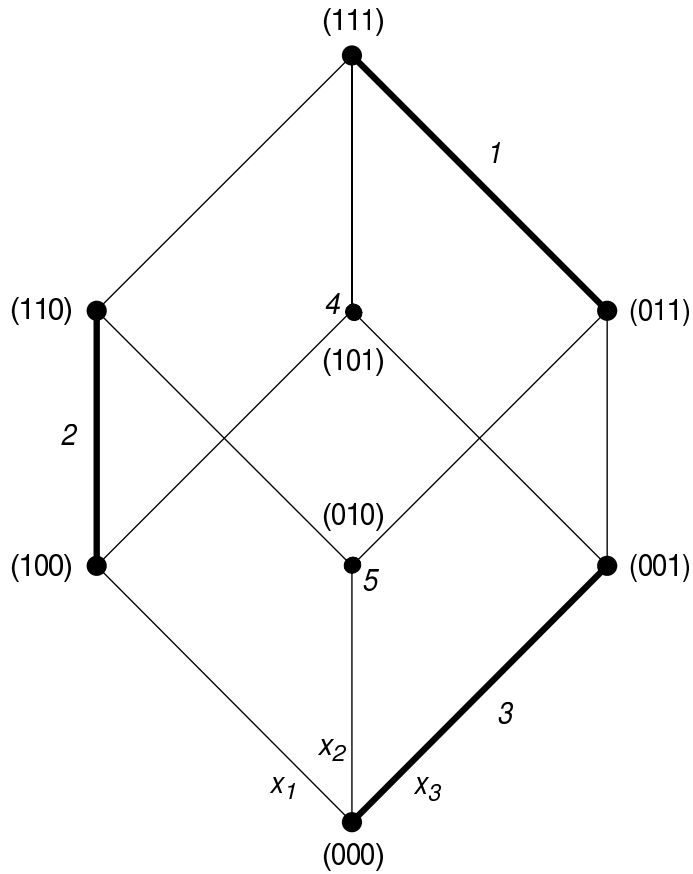

Рис. 1. Тематическое разбиение куба $B^{3}$

Тогда функция

$$
f\left(y_{1}, \ldots, y_{30}\right)= \begin{cases}1 & \text { при } y_{8}=1, y_{27}=1 ; \\ 2 & \text { при } y_{6}=1, y_{27}=0 ; \\ 3 & \text { при } y_{6}=0, y_{8}=0 ; \\ 4 & \text { при } y_{6}=1, y_{8}=0, y_{27}=1 ; \\ 5 & \text { при } y_{6}=0, y_{8}=1, y_{27}=0 ;\end{cases}
$$

является примером темоопределяющей функции над $R_{0}$ (здесь $q_{1}=6, q_{2}=8, q_{3}=27$ и $y_{6}, y_{8}, y_{27}$ - тематические переменные).

Понятно, что для любой ТОФ $f$ множество ее существенных переменных совпадает с множеством ее тематических переменных.

Через $\Phi_{R}^{n}$ обозначим множество в точности всех ТОФ от $n$ переменных над разбиением $R$. Поскольку при фиксации $R$ ТОФ однозначно определяется ее основанием, справедливо равенство $\left|\Phi_{R}^{n}\right|=A_{n}^{k}$, где $k$ - размерность тематического $R$-куба, $A_{n}^{k}-$ число размещений из $n$ по $k$.

Пусть теперь задано некоторое тематическое разбиение $R$ и задан оператор $A_{f}$, вычисляющий для произвольного набора из $B^{n}$ значение некоторой ТОФ $f \in \Phi_{R}^{n}$ на этом наборе. Задача данной работы - за минимальное число обращений к оператору $A_{f}$ полностью восстановить таблицу значений функции $f\left(y_{1}, \ldots, y_{n}\right)$. Эту задачу назовем задачей определения ТОФ $f$ по разбиению $R$ и оператору $A_{f}$.

Рассмотрим множество $\mathscr{F}$ алгоритмов, решающих указанную задачу для любого разбиения $R \in \mathscr{R}_{k}$ и для любой ТОФ $f \in \Phi_{R}^{n}$, где $\mathscr{R}_{k}-$ множество всех тематических 
разбиений куба $B^{k}$. На вход любого алгоритма $F$ из этого множества подаются разбиение $R \in \mathscr{R}_{k}$ и оператор $\mathscr{A}_{f}$. На выходе $F$ выдает таблицу значений ТОФ $f$ или, что то же самое, номера $k$ искомых тематических переменных. Другими словами, при фиксированном тематическом разбиении $R$ для произвольной ТОФ $f \in \Phi_{R}^{n}$ любой алгоритм $F \in \mathscr{F}$ с помощью оператора $A_{f}$ полностью восстанавливает таблицу значений ТОФ $f$. Работа алгоритма $F$ заключается в том, что он последовательно запрашивает значения оператора $A_{f}$ на наборах из $B^{n}$. При этом алгоритм $F$ предполагается условным, то есть при выборе очередного набора он может пользоваться знаниями о значениях функции на ранее поданных им наборах. Любой тройке, алгоритму $F$, тематическому разбиению $R \in \mathscr{R}_{k}$ и ТОФ $f \in \Phi_{R}^{n}$, можно сопоставить число $\varphi(F, R, n, f)$, равное числу обращений к оператору $A_{f}$ в процессе восстановления таблицы значений функции $f$ с помощью алгоритма $F$. Введем обозначения

$$
\begin{aligned}
\varphi(F, R, n) & =\max _{f \in \Phi_{R}^{n}} \varphi(F, R, n, f), \\
\varphi(F, k, n) & =\max _{R \in \Re_{k}} \varphi(F, R, n), \\
\varphi(k, n) & =\min _{F \in \mathscr{F}} \varphi(F, k, n) .
\end{aligned}
$$

Целью настоящей работы является описание асимптотического поведения функции $\varphi(k, n)$ при $n \rightarrow \infty$.

Очевидно, что для любого разбиения $R$ и для любой $f \in \Phi_{R}^{n}$ алгоритм, запрашивающий значения оператора $A_{f}$ на всех $2^{n}$ наборах, позволяет полностью определить $f$. Отсюда следует тривиальная верхняя оценка $\varphi(k, n) \leqslant 2^{n}$.

В работе доказана следующая теорема.

Теорема 1. Если $n \rightarrow \infty, k \rightarrow \infty$ так, что $k \leqslant c n$, где $c<1$, то

$$
\varphi(k, n) \sim k \log _{2} n .
$$

\section{3. Нижняя оценка}

Докажем сначала нижнюю оценку для одномерного тематического куба. Здесь существует единственное возможное разбиение куба $x_{1} \vee \bar{x}_{1}$. Для удобства изложения будем считать в одномерном случае, что тема $\bar{x}_{1}$ имеет номер 0 , а тема $x_{1}$ имеет номер 1 . Существует $n$ возможных ТОФ, подходящих под это разбиение. Докажем, что для любого алгоритма найдется некоторая плохая функция $f$ из этих $n$, для определения которой алгоритм запросит значения оператора $\mathscr{A}_{f}$ минимум на ] $\log _{2} n$ [ наборах.

Итак, фиксируем произвольный алгоритм $G_{0}$. Шагом алгоритма $G_{0}$ будем считать запрос значения оператора $\mathscr{A}_{f}$ на некотором наборе. Пусть $a_{i}-$ набор, который сгенерирован алгоритмом $G_{0}$ на $i$-м шаге, $a_{i}^{j}-j$-я компонента этого набора, $i \in\{1,2, \ldots\}$, $j \in\{1, \ldots, n\}$. На каждом $i$-м шаге будем строить множество $T_{i}=\left\{y_{j_{1}}, \ldots, y_{j_{i}}\right\}$, $j_{i} \in\{1, \ldots, n\}$, переменных, которые все еще могут быть тематической переменной. Для любых $j \in\{1, \ldots, n\}, i \in\{1,2, \ldots\}$, переменная $y_{j}$ лежит в множестве $T_{i}$ тогда и только тогда, когда, $\mathscr{A}_{f}\left(a_{s}\right)=a_{s}^{j}$ для всех $s \in\{1, \ldots, i\}$. Положим $T_{0}=\left\{y_{1}, \ldots, y_{n}\right\}$. Очевидно, $T_{0} \supseteq T_{1} \supseteq T_{2} \supseteq \ldots$ Очевидно также, что алгоритм $G_{0}$ решает задачу нахождения тематической переменной на $s$-м шаге тогда и только тогда, когда $\left|T_{s}\right|=1$. Для любого 
$i \in\{1,2, \ldots\}$, если $T_{i-1}=\left\{y_{j_{1}}, \ldots, y_{j_{i-1}}\right\}$, то положим $a_{i}^{\prime}=\left(a_{i}^{j_{1}}, \ldots, a_{i}^{j_{i-1}}\right)$ и

$$
z\left(a_{i}\right)= \begin{cases}0, & \text { если в наборе } a_{i}^{\prime} \text { нулей больше, чем единиц, } \\ 1 & \text { в противном случае. }\end{cases}
$$

Построим для алгоритма $G_{0}$ необходимую функцию $f$. Заметим, что до тех пор, пока мы не определили тематическую переменную, значение оператора на произвольном наборе мы можем выбирать произвольным образом. На каждом $i$-м шаге, $i \in\{1,2, \ldots\}$, полагаем $\mathscr{A}_{f}\left(a_{i}\right)=z\left(a_{i}\right)$. При указанном способе выбора значений оператора $\mathscr{A}_{f}$ множеству $T_{i}, i \in\{1,2, \ldots\}$, принадлежит не менее половины переменных множества $T_{i-1}$. Значит, потребуется минимум $s=] \log _{2} n$ [ наборов для того, чтобы мощность множества $T_{s}$ была единицей. Это и завершает доказательство.

В качестве иллюстрации сказанного приведем следующий пример:

$\begin{array}{lcccccccll} & y_{1} & y_{2} & y_{3} & y_{4} & y_{5} & y_{6} & y_{7} & & \\ a_{1} & 1 & 1 & 1 & 0 & 1 & 1 & 1 & A_{f}\left(a_{1}\right)=1, & T_{1}=\left\{y_{1}, y_{2}, y_{3}, y_{5}, y_{6}, y_{7}\right\}, \\ a_{2} & 0 & 1 & 0 & 1 & 1 & 1 & 0, & \mathscr{A}_{f}\left(a_{2}\right)=1, & T_{2}=\left\{y_{2}, y_{5}, y_{6}\right\}, \\ a_{3} & 1 & 0 & 1 & 0 & 1 & 0 & 1 & \mathscr{A}_{f}\left(a_{3}\right)=0, & T_{3}=\left\{y_{2}, y_{6}\right\} .\end{array}$

Здесь $n=7$, и после подачи $] \log _{2} n\left[=3\right.$ наборов переменные $y_{2}$ и $y_{6}$ все еще могут быть тематической переменной.

Итак, $\varphi(1, n) \geqslant] \log _{2} n[$. На самом деле, как мы покажем в следующем разделе, $\varphi(1, n)=] \log _{2} n[$. Перейдем к $k$-мерному случаю. В нем разбиений уже много, как и ТОФ, удовлетворяющих этим разбиениям. Поэтому при выборе самой плохой ситуации у нас есть возможность варьировать и разбиение, и функцию.

Лемма 1. Справедливо неравенство

$$
\left.\varphi(k, n) \geqslant(k-] \log _{2} k[)\right] \log _{2}(n-k+1)[.
$$

Доказательство. Построим разбиение, зависящее от переменных $x_{1}, \ldots, x_{k}$. Положим $m=] \log _{2} k\left[\right.$. Пусть $m$-мерный булев куб $B^{m}$ задается переменными $x_{1}, \ldots, x_{m}$. Положим $R_{j}=B_{\sigma_{1}, \ldots, \sigma_{m}}^{m, 1, \ldots, m}$, где

$$
\left\|\left(\sigma_{1}, \ldots, \sigma_{m}\right)\right\|=\sum_{i=1}^{m} 2^{m-i} \sigma_{i}=j .
$$

Для куба $B^{m}$ рассмотрим тематическое разбиение $R^{\prime}=\left\{R_{j}, j=0,1, \ldots, 2^{m}-1\right\}$, которое состоит из $2^{m}$ тем, причем каждая тема содержит только одну вершину $m$-мерного куба. Рассмотрим теперь разбиение $R$ булева $k$-мерного куба $B^{k}$

$$
R=\left\{R_{0}^{0}, R_{0}^{1}, \ldots, R_{k-m-1}^{0}, R_{k-m-1}^{1}, R_{k-m}, \ldots, R_{2^{m}-1}\right\},
$$

где для каждого $j \in\{0,1, \ldots, k-m-1\}$ подкубы $R_{j}^{0}$ и $R_{j}^{1}$ булева $k$-мерного куба определяются конъюнкциями $x_{1}^{\sigma_{1}} \ldots x_{m}^{\sigma_{m}} \bar{x}_{j+m+1}$ и $x_{1}^{\sigma_{1}} \ldots x_{m}^{\sigma_{m}} x_{j+m+1}$ соответственно, где $\left\|\left(\sigma_{1}, \ldots, \sigma_{m}\right)\right\|=j$, а каждый подкуб $R_{j}$, как и раньше, определяется конъюнкцией $x_{1}^{\sigma_{1}} \ldots x_{m}^{\sigma_{m}}$, где $\left\|\left(\sigma_{1}, \ldots, \sigma_{m}\right)\right\|=j, j \in\left\{k-m, \ldots, 2^{m}-1\right\}$. Очевидно, что построенное разбиение $R$ является тематическим разбиением $k$-мерного куба, зависящим от переменных $x_{1}, \ldots, x_{k}$.

Для произвольного алгоритма $G_{0}$ построим плохую темоопределяющую функцию, то есть функцию, которую $G_{0}$ не сможет определить, запросив значения оператора $\mathscr{A}_{f}$ 
на менее чем $(k-m)] \log _{2}(n-k+1)\left[\right.$ наборах. Пусть $g: B^{n} \rightarrow\{1, \ldots,|R|\}-$ некоторая функция, $C \subseteq B^{n}-$ некоторое подмножество $n$-мерного куба. Тогда положим

$$
\Phi_{g, C, R}^{n}=\left\{f \in \Phi_{R}^{n}:\left.f\right|_{C}=\left.g\right|_{C}\right\},
$$

где через $\left.f\right|_{C}$ обозначено сужение функции $f$ на множество $C$. Пусть также для произвольного набора $a \in B^{n}$ и $m \in\{1, \ldots,|R|\}$

$$
\Phi_{g, C, R, a, m}^{n}=\left\{f \in \Phi_{g, C, R}^{n}: f(a)=m\right\} .
$$

Шагом алгоритма $G_{0}$, как и раньше, будем считать запрос значения оператора $A_{f}$ на некотором наборе. Пусть $a_{i}-$ набор, сгенерированный алгоритмом $G_{0}$ на $i$-м шаге, $a_{i}^{j}-j$-я компонента этого набора, $i \in\{1,2, \ldots\}, j \in\{1, \ldots, n\}$. По аналогии с одномерным случаем введем множество $T_{i, j} \in\left\{y_{1}, \ldots, y_{n}\right\}$ переменных, которые могут быть переменной $x_{j}$ после $i$-го шага алгоритма $G_{0}$. Положим $T_{0, j}=\left\{y_{1}, \ldots, y_{n}\right\}$. Очевидно, что алгоритм $G_{0}$ решает задачу нахождения тематических переменных на $s$-м шаге тогда и только тогда, когда $\left|T_{s, j}\right|=1$ для любого $j \in\{1, \ldots, k\}$. Для любых $i \in\{1,2, \ldots\}, j \in\{1, \ldots, k\}$, если $T_{i-1, j}=\left\{y_{j_{1}}, \ldots, y_{j_{i-1}}\right\}$, то положим $a_{i, j}^{\prime}=\left(a_{i}^{j_{1}}, \ldots, a_{i}^{j_{i-1}}\right)$ и

$$
z^{j}\left(a_{i}\right)= \begin{cases}0, & \text { если в наборе } a_{i, j}^{\prime} \text { нулей больше, чем единиц, } \\ 1 & \text { в противном случае. }\end{cases}
$$

Пусть $C_{l-1}=\left\{a_{1}, \ldots, a_{l-1}\right\}-$ упорядоченное множество наборов, поданных алгоритмом $G_{0}$ за первые $l-1$ шагов. Предположим, что мы уже задали значения $\mathscr{A}_{f}$ на этих $l-1$ наборах. Рассмотрим функцию $g: C_{l-1} \rightarrow\{1, \ldots,|R|\}$ такую, что $g\left(a_{i}\right)=\mathscr{A}_{f}\left(a_{i}\right)$, $i=1, \ldots, l-1$. Пусть $a_{l}-$ набор, поданный алгоритмом $G_{0}$ на $l$-м шаге. Значение $\mathscr{A}_{f}\left(a_{l}\right)$ выберем следующим образом. Если существует такой номер $j \in\left\{k-m, \ldots, 2^{m}-1\right\}$, что $\Phi_{g, C, R, a_{l}, \mathcal{N}\left(R_{j}\right)}^{n} \neq \varnothing$, то полагаем $\mathscr{A}_{f}\left(a_{l}\right)=\mathcal{N}\left(R_{j}\right)$. В противном случае обязательно существует такой номер $j \in\{0,1, \ldots, k-m-1\}$, что $\Phi_{g, C, R, a_{l}, \mathcal{N}\left(R_{j}^{0}\right)}^{n} \neq \varnothing$ (отсюда, очевидно, и $\left.\Phi_{g, C, R, a_{l}, \mathcal{N}\left(R_{j}^{1}\right)}^{n} \neq \varnothing\right)$. Тогда полагаем

$$
\mathscr{A}_{f}\left(a_{l}\right)= \begin{cases}\mathcal{N}\left(R_{j}^{0}\right), & \text { если } z^{j+m+1}\left(a_{l}\right)=0 \\ \mathcal{N}\left(R_{j}^{1}\right), & \text { если } z^{j+m+1}\left(a_{l}\right)=1\end{cases}
$$

Рассмотрим на некотором $l$-м шаге алгоритма $G_{0}$ для фиксированного номера $j \in\{0,1, \ldots, k-m-1\}$ все наборы $a_{i_{1}}, \ldots, a_{i_{s}}, i_{s} \in\{1, \ldots, l\}$, лежащие либо в теме $R_{j}^{0}$, либо в теме $R_{j}^{1}$ (в том же порядке, в котором их генерировал алгоритм). Тогда при указанном выше способе выбора значений оператора $\mathscr{A}_{f}$ множеству $T_{i_{r}, j+m+1}$, $r \in\{1, \ldots, s\}$, принадлежит не менее половины переменных множества $T_{i_{r-1}, j+m+1}$. Значит, для определения переменной $x_{j+m+1}$ алгоритм $G_{0}$ сгенерирует в худшем случае минимум ] $\log _{2}(n-k+1)$ [ наборов, лежащих в одной из тем $R_{j}^{0}$ или $R_{j}^{1}$ (так как ни в одной другой теме переменная $x_{j+m+1}$ не встречается). Отсюда, для определения всех переменных $x_{j+m+1}, j \in\{0,1, \ldots, k-m-1\}$, потребуется не менее $\left.(k-m)\right] \log _{2}(n-k+1)[$ наборов, что и завершает доказательство в многомерном случае.

\section{4. Верхняя оценка}

Снова начнем с одномерного случая. Напомним, что в одномерном случае существует единственное возможное тематическое разбиение $R$ одномерного булева куба $x_{1} \vee \bar{x}_{1}$ и $n$ 
возможных ТОФ, подходящих под это разбиение (то есть $\left|\Phi_{R}^{n}\right|=n$ ). Построим алгоритм, определяющий любую из этих $n$ функций за ] $\log _{2} n$ [ запросов значений соответствующего оператора.

Упорядоченное множество наборов $A$ из $B^{n}$ назовем правильным, если в матрице, строками которой являются эти наборы, все $n$ столбцов различны. Эту матрицу также будем обозначать $A$. Очевидно, существует правильное множество мощности $] \log _{2} n[$,

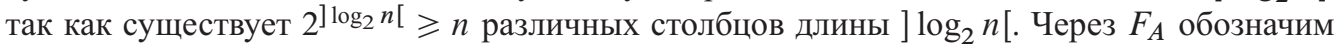
алгоритм, который последовательно запрашивает значения оператора $\mathscr{A}_{f}$ на всех строках $\left\{a_{1}, \ldots, a_{|A|}\right\}$ матрицы $A$.

Лемма 2. Если $R$ - тематическое разбиение одномерного куба, то для любой функции $f \in \Phi_{R}^{n}$ и для любого правильного множества $A$ мощности $] \log _{2} n[$ справедливо неравенство

$$
\left.\varphi\left(F_{A}, R, n, f\right) \leqslant\right] \log _{2} n[.
$$

Доказательство. Пусть $c=\left(c_{1}, \ldots, c\right] \log _{2} n[)$, где

$$
c_{s}= \begin{cases}0, & \text { если } \mathscr{A}_{f}\left(a_{s}\right)=\mathcal{N}\left(\bar{x}_{1}\right), \\ 1, & \text { если } \mathscr{A}_{f}\left(a_{s}\right)=\mathcal{N}\left(x_{1}\right),\end{cases}
$$

$s \in\{1, \ldots,] \log _{2} n[\}$. Так как искомая тематическая переменная существует, в матрице, образованной наборами множества $A$, найдется столбец $b_{l}=c, l \in\{1, \ldots, n\}$. По определению правильного множества такой столбец единствен. Легко видеть, что переменная $y_{l}$ и является искомой тематической.

Для 7 переменных правильное множество может выглядеть, например, следующим образом:

$$
\left(\begin{array}{lllllll}
1 & 1 & 1 & 1 & 0 & 0 & 0 \\
1 & 1 & 0 & 0 & 1 & 1 & 0 \\
1 & 0 & 1 & 0 & 1 & 0 & 1
\end{array}\right) .
$$

В силу произвольности $f$ из леммы 1 следует неравенство $\varphi(1, n) \leqslant] \log _{2} n[$. Итак, для одномерного случая справедлива точная оценка $\varphi(1, n)=] \log _{2} n[$.

Перейдем к $k$-мерному случаю. Напомним, что фиксирован некоторый $k$-мерный тематический $R$-куб, где элементы $R$ - элементарные конъюнкции (темы) вида $x_{i_{1}}^{\sigma_{1}} \ldots x_{i_{t_{j}}}^{\sigma_{t_{j}}}$, $t_{j}<k, j \in\{1, \ldots,|R|\}$. Назовем тему тематического разбиения крайней, если все переменные входят в нее без отрицаний, либо все переменные входят в нее с отрицаниями. Очевидно, в любом тематическом разбиении существуют ровно 2 крайних темы.

Рассмотрим ДНФ, описывающую тематическое разбиение $R$ булева $k$-мерного куба от переменных $x_{1}, \ldots, x_{k}$. Положим $x_{i_{1}}=0, \ldots, x_{i_{l}}=0, x_{j_{1}}=1, \ldots, x_{j_{r}}=1$, $\left\{i_{1}, \ldots, i_{l}\right\} \cap\left\{j_{1}, \ldots, j_{r}\right\}=\varnothing, l, r \in\{1, \ldots, k\}, l+r<k$. Упростим имеющуюся ДНФ, воспользовавшись тождествами $x \cdot 1=x, x \cdot 0=0$. В результате получим ДНФ, описывающую некоторое новое тематическое разбиение $R^{\prime}$ куба, размерность которого не превышает $k-(l+r)$. Будем называть разбиение $R^{\prime}$ разбиением, полученным из $R$ фиксацией переменных $x_{i_{1}}, \ldots, x_{i_{l}}$ нулями и переменных $x_{j_{1}}, \ldots, x_{j_{r}}$ единицами.

Далее для фиксированного разбиения $R$ и ТОФ $f$ под значением оператора $\mathscr{A}_{f}$ на некотором наборе будем понимать не номер элементарной конъюнкции, задающей тему, в которой этот набор лежит, а саму элементарную конъюнкцию. Пусть теперь вместо $R$ мы имеем разбиение $R^{\prime}$, полученное из $R$ фиксацией переменных $x_{i_{1}}, \ldots, x_{i_{l}}$ нулями 
и переменных $x_{j_{1}}, \ldots, x_{j_{r}}$ единицами. Тогда для произвольного набора $a$ преобразуем конъюнкцию $\mathscr{A}_{f}(a)$, фиксировав $x_{i_{1}}, \ldots, x_{i_{l}}$ нулями и $x_{j_{1}}, \ldots, x_{j_{r}}$ единицами. Если из контекста ясно, о каком разбиении идет речь, то эту конъюнкцию будем также обозначать $\mathscr{A}_{f}(a)$.

Пусть $a=\left(a_{1}, \ldots, a_{n}\right)$ - некоторый набор из $B^{n}, M-$ некоторое множество номеров, не превосходящих $n$. Рассмотрим набор $b=\left(b_{1}, \ldots, b_{n}\right) \in B^{n}$ такой, что

$$
b_{i}= \begin{cases}a_{i}, & i \in M, \\ \bar{a}_{i}, & i \notin M,\end{cases}
$$

$i \in\{1, \ldots, n\}$. Будем обозначать набор $b$ через $\neg^{M}(a)$.

Опишем алгоритм $F_{0}$ такой, что для любых $R \in \mathscr{R}_{k}, f \in \Phi_{R}^{n}$ справедливо неравенство

$$
\left.\varphi\left(F_{0}, R, n, f\right) \leqslant 2 k\right] \log _{2} n[+k .
$$

Рассмотрим некоторую матрицу $A$. Считаем, что ее строки и столбцы можно помечать, пусть $m_{1}-$ массив номеров помеченных строк, $m_{2}-$ массив номеров помеченных столбцов. Через $\mathscr{F}=\left(\mathscr{F}_{1}, \ldots, \mathscr{F}_{k}\right)$ обозначим вектор длины $k$ такой, что

$$
\mathscr{f}_{j}= \begin{cases}l, & \text { если } \Psi\left(x_{j}\right)=y_{l}, \\ *, & \text { если значение } \Psi\left(x_{j}\right) \text { неизвестно, }\end{cases}
$$

$j \in\{1, \ldots, k\}, l \in\{1, \ldots, n\}$. Вектор $\mathscr{F}$ назовем ответом. Будем называть переменную $x_{j}$, $j \in\{1, \ldots, k\}$, найденной, если $\mathscr{F}_{j} \neq *$.

В основе алгоритма $F_{0}$ лежит рекурсивная процедура $\operatorname{TSEARCH}\left(A, R, m_{1}, m_{2}\right)$. Опишем ее работу. Последовательно вычисляем значения оператора $A_{f}$ на непомеченных строках матрицы $A$ (пусть это строки $a_{1}, \ldots, a_{d}$ ). Возможны 2 случая.

1. Мы находим строку $a_{i}, i \in\left\{a_{1}, \ldots, a_{d}\right\}: \mathscr{A}_{f}\left(a_{i}\right)$ - некрайняя тема. Назовем $a_{i}$ разделяющим набором, а $\mathscr{A}_{f}\left(a_{i}\right)$ - разделяющей темой, пусть эта тема задается конъюнкцией $x_{i_{1}} \ldots x_{i_{r}} \bar{x}_{i_{r+1}} \ldots \bar{x}_{i_{t}}$.

Рассмотрим все столбцы $b_{i_{1}}, \ldots, b_{i_{s}}$ матрицы $A$, пересечения которых со строкой $a_{i}$ - нули. Определим новую матрицу $A^{\prime}=\left(b_{1}^{\prime}, \ldots, b_{n}^{\prime}\right)$, где для $j \in\{1, \ldots, n\}$

$$
\begin{cases}b_{j}^{\prime}=b_{j}, & \text { если } j \neq i_{1}, \ldots, i_{s}, \\ b_{j}^{\prime}-\text { нулевой столбец } & \text { в противном случае. }\end{cases}
$$

Пометим строку $a_{i}^{\prime}$, положив $m_{1}^{\prime}=m_{1} \cup\{i\}$, и столбцы $b_{i_{1}}^{\prime}, \ldots, b_{i_{s}}^{\prime}$, положив $m_{2}^{\prime}=m_{2} \cup\left\{i_{1}, \ldots, i_{s}\right\}$. Рассмотрим разбиение $R^{\prime}$, полученное из $R$ фиксацией переменных $x_{i_{r+1}}, \ldots, x_{i_{t}}$ нулями. Вызываем процедуру $\operatorname{TSEARCH}\left(A^{\prime}, R^{\prime}, m_{1}^{\prime}, m_{2}^{\prime}\right)$.

Теперь рассмотрим все столбцы $b_{l_{1}}, \ldots, b_{l_{h}}, h \in\{1, \ldots, n\}$, матрицы $A$, пересечения которых со строкой $a_{i}$ - единицы. Определим новую матрицу $A^{\prime \prime}=\left(b_{1}^{\prime \prime}, \ldots, b_{n}^{\prime \prime}\right)$, где для $j \in\{1, \ldots, n\}$

$$
\begin{cases}b_{j}^{\prime \prime}=b_{j}, & \text { если } j \neq l_{1}, \ldots, l_{h}, \\ b_{j}^{\prime \prime}-\text { единичный столбец } & \text { в противном случае. }\end{cases}
$$

Пометим строку $a_{i}^{\prime \prime}$, положив $m_{1}^{\prime \prime}=m_{1} \cup\{i\}$, и столбцы $b_{l_{1}}^{\prime \prime}, \ldots, b_{l_{h}}^{\prime \prime}$, положив $m_{2}^{\prime \prime}=m_{2} \cup\left\{l_{1}, \ldots, l_{h}\right\}$. Рассмотрим разбиение $R^{\prime \prime}$, полученное из разбиения $R$ фиксацией переменных $x_{i_{1}}, \ldots, x_{i_{r}}$ единицами. Полученное разбиение обозначим $R^{\prime \prime}$. Вызываем процедуру TSEARCH $\left(A^{\prime \prime}, R^{\prime \prime}, m_{1}^{\prime \prime}, m_{2}^{\prime \prime}\right)$. 
2. Для любого $i \in\{1, \ldots, d\}$ тема $\mathscr{A}_{f}\left(a_{i}\right)$ - одна из двух крайних тем. Считаем, что среди непомеченных строк матрицы $A$ есть и лежащие в отрицательной крайней теме, и в положительной. Иначе полагаем $a_{1}=\neg^{m_{2}}\left(a_{1}\right)$, вычисляем $\mathscr{A}_{f}\left(a_{1}\right)$. При этом строка $a_{1}$ уже не обязана лежать в крайней теме. Берем первую переменную $x_{j}, j \in\{1, \ldots, k\}$, которая входит во все темы $\mathscr{A}_{f}\left(a_{i}\right), i \in\{1, \ldots, d\}$. Если такой переменной не существует, то выдаем ошибку и заканчиваем работу. Пусть $c=\left(c_{1}, \ldots, c_{d}\right)$, где

$$
c_{s}= \begin{cases}0, & \text { если в } \mathscr{A}_{f}\left(a_{s}\right) x_{j} \text { входит с отрицанием, } \\ 1, & \text { если в } \mathscr{A}_{f}\left(a_{s}\right) x_{j} \text { входит без отрицания, }\end{cases}
$$

$s \in\{1, \ldots, d\}$. Среди непомеченных столбцов матрицы, образованной строками $a_{1}, \ldots, a_{d}$ находим столбец $b_{l}=c, l \in\{1, \ldots, n\}$. Переменная $y_{l}$ является тематической. Помещаем ее в ответ, полагая $\mathscr{f}_{j}=l$.

Собственно алгоритм $F_{0}$ выглядит следующим образом.

(1) Устанавливаем $\mathscr{g}=(*, \ldots, *), R^{\prime}=R, n^{\prime}=n$. Пусть $A-$ матрица $] \log _{2} n[\times n$, соответствующая произвольному правильному множеству. Полагаем $A^{\prime}=A$.

(2) Вызываем процедуру $\operatorname{TSEARCH}\left(A^{\prime}, R^{\prime}, \varnothing, \varnothing\right)$.

(3) Если в векторе ответа $\mathscr{E}$ все компоненты не равны $*$, то есть определились, то алгоритм $F_{0}$ завершает работу.

(4) Фиксируем найденные переменные таким образом, чтобы разбиение $R^{\prime}$, полученное из $R$ операцией фиксации этих переменных, зависело хотя бы от одной переменной. Такая фиксация обязательно существует.

(5) Пусть $Q=\left\{q_{i_{1}}, \ldots, q_{i_{l}}\right\}, l<k,-$ множество номеров всех найденных тематических переменных. Положим $n^{\prime}=n-l$. Фиксируем произвольное правильное множество с матрицей $A^{\prime}$ размера $] \log _{2} n^{\prime}\left[\times n^{\prime}\right.$. Нумеруем ее столбцы числами из множества $\{1, \ldots, n\} \backslash Q$. Пусть матрица $A^{\prime \prime}$ получена из матрицы $A^{\prime}$ добавлением $l$ столбцов, имеющих в матрице $A^{\prime \prime}$ номера $q_{i_{1}}, \ldots, q_{i_{l}}$. Причем столбец матрицы $A^{\prime \prime}$ с номером $q_{i_{p}}, p \in\{1, \ldots, l\},-$ единичный, если переменная $x_{i_{p}}$ фиксирована единицей, и нулевой, если $x_{i_{p}}$ фиксирована нулем. Под значением оператора $\mathscr{A}_{f}$ на некоторой строке матрицы $A^{\prime}$ будем понимать значение $\mathscr{A}_{f}$ на строке матрицы $A^{\prime \prime}$ с тем же номером.

(6) Идем на шаг 2.

Итак, алгоритм $F_{0}$ есть, по сути, некоторое конечное число вызовов процедуры TSEARCH. Согласно приведенному выше описанию этой функции, при фиксированных $n, R \in \mathscr{R}_{k^{\prime}}, f \in \Phi_{R}^{n}$ и правильном множестве $A$ работу процедуры TSEARCH $(A, R, \varnothing, \varnothing)$ можно описывать бинарным деревом $\mathscr{D}_{n, R, f, A}$. В каждой неконцевой вершине этого дерева запрашиваются значения оператора $\mathscr{A}_{f}$ на строках некоторой матрицы до тех пор, пока не найден разделяющий набор, лежащий в некрайней разделяющей теме. Затем в зависимости от фиксации переменных разделяющей темы нулями или единицами происходит переход по нулю к левому потомку рассматриваемой вершины и по единице к правому потомку соответственно. На нулевом уровне (в корне дерева $\mathscr{D}_{n, R, f, A}$ ) запрашивается исходная матрица $A$. Матрицу, запрошенную в некоторой вершине $v i$-го уровня дерева $\mathscr{D}_{n, R, f, A}$, обозначим через $A^{\sigma_{1}, \ldots, \sigma_{i}}$, если для любого $j, j \in\{0,1, \ldots, i-1\}$, в вершине $w$ $j$-го уровня, потомком которой является вершина $v$, происходит переход по $\sigma_{j}$ к прямому 
потомку $w^{\prime}$, причем $v$ является потомком $w^{\prime}$. В дальнейшем вершину $v$ будем обозначать $v^{\sigma_{1}, \ldots, \sigma_{i}}$, тематическое разбиение, заданное в вершине $v$, будем обозначать $R^{\sigma_{1}, \ldots, \sigma_{i}}$, а число переменных, от которых зависит $R^{\sigma_{1}, \ldots, \sigma_{i}}$, равно $k^{\sigma_{1}, \ldots, \sigma_{i}}$.

Перед тем, как перейти к проверке корректности работы алгоритма, приведем пример его работы на разбиении $R_{0}$, изображенном на рис. 1 и задаваемом соотношением (1). Пусть $n=30$, и пусть оператор $\mathscr{A}_{f}$ выдает по набору из $B^{30}$ значение ТОФ, определяемой соотношением (2). При этом, естественно, ТОФ $f$ алгоритм $F_{0}$ не знает. Задача алгоритма $F_{0}$ как раз и состоит в определении $f$ по заданному тематическому разбиению $R_{0}$ и значениям оператора $A_{f}$ на наборах из $B^{30}$.

Рассмотрим множество наборов, значения оператора $A_{f}$ на которых запрашивает алгоритм $F_{0}$ для определения ТОФ $f\left(x_{1}, \ldots, x_{30}\right)$ :

$A$ Исходное разбиение $x_{2} x_{3} \vee x_{1} \bar{x}_{3} \vee \bar{x}_{1} \bar{x}_{2} \vee x_{1} \bar{x}_{2} x_{3} \vee \bar{x}_{1} x_{2} \bar{x}_{3}$

222222222222222222222222222222

$000000000000000111111111111111 \in \bar{x}_{1} \bar{x}_{2}$ $000000001111111000000001111111 \in \bar{x}_{1} \bar{x}_{2}$ $000011110000111000011110000111 \in x_{1} \bar{x}_{3}$

$A^{0}$ Фиксируем $x_{3}$ нулем, получаем разбиение $x_{1} \vee \bar{x}_{1} \bar{x}_{2} \vee \bar{x}_{1} x_{2}$ 000022220000222000022220000222

$000000000000000000011110000111 \in \bar{x}_{1} \bar{x}_{2}$ $00000000000011100000000000000111 \in \bar{x}_{1} \bar{x}_{2}$ $000000110000001000000110000001 \in \bar{x}_{1} x_{2}$

$A^{00}$ Фиксируем $x_{1}$ нулем, получаем разбиение $\bar{x}_{2} \vee x_{2}$ 000000220000002000000220000002 $00000000000000000000000000111000000001 \in \bar{x}_{2}$ $0000000000000001000000000000000001 \in \bar{x}_{2}$ $000000010000000000000010000000 \in x_{2}$

Очевидно, подходит только 8-й столбец $\Rightarrow \Psi\left(x_{2}\right)=y_{8}$

$A^{01}$ Фиксируем $x_{2}$ единицей, получаем разбиение $x_{1} \vee \bar{x}_{1}$ 000022110000221000022110000221

$0000000110000000100001111100000111 \in \bar{x}_{1}$ $00000001100000111100000001100000111 \in \bar{x}_{1}$ $000001110000011000001110000011 \in x_{1}$

Очевидно, подходит только 6-й столбец $\Rightarrow \Psi\left(x_{1}\right)=y_{6}$

$A^{1}$ Фиксируем $x_{1}$ единицей, получаем разбиение $x_{2} x_{3} \vee \bar{x}_{3} \vee \bar{x}_{2} x_{3}$ (переменная $x_{2}$ стала константой 1)

222211112222111222211112222111

$000011110000111111111111111111 \in x_{2} x_{3}$ $000011111111111000011111111111 \in x_{2} x_{3}$ $001111110011111001111110011111 \in x_{2} x_{3}$ $010111110101111010111110101111 \in x_{2} x_{3}$ Очевидно, подходит только 27-й столбец $\Rightarrow \Psi\left(x_{2}\right)=y_{27}$, или $\Psi\left(x_{3}\right)=y_{27} \Rightarrow$ согласно алгоритму, обращаем первый набор, получаем новую матрицу $A^{1}$ :

222211112222111222211112222111

$111111111111111000011110000111 \in \bar{x}_{3}$

$A^{1} \quad 000011111111111000011111111111 \in x_{2} x_{3}$ $001111110011111001111110011111 \in x_{2} x_{3}$ $010111110101111010111110101111 \in x_{2} x_{3}$ 


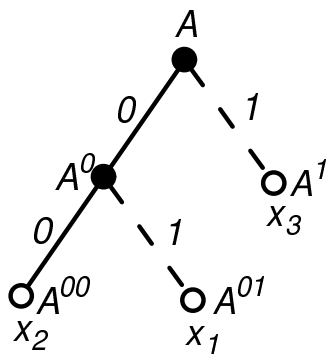

Рис. 2. Дерево $\mathscr{D}_{30, R_{0}, f, A}$

Теперь очевидно, что именно $\Psi\left(x_{3}\right)=y_{27}$.

Здесь каждому набору приписана тема, в которой он лежит (или, что то же, значение оператора $\mathscr{A}_{f}$ на этом наборе). Каждая таблица начинается с вспомогательного набора длины 30, элементы которого принимают значения из $\{0,1,2\}: i$-й элемент этого набора равен 0 , если $y_{i}$ фиксирована на данном этапе нулем, равен 1 , если $y_{i}$ фиксирована единицей и равен 2 , если $y_{i}$ пока не фиксирована (в терминах, использованных при описании алгоритма, столбцу соответствует цифра 2, если он не является помеченным). Остальные наборы каждой таблицы являются строками текущей матрицы $A$ (в зависимости от шага алгоритма, они названы $A, A^{0}, A^{1}, A^{00}, A^{01}$ ). Для удобства восприятия из таблиц убраны все незначимые наборы матрицы $A$, а именно, помеченные наборы и наборы, идущие в матрице $A$ после разделяющего набора. Бинарное дерево $\mathscr{D}_{30, R_{0}, f, A}$ изображено на рис. 2.

Из значений оператора $\mathscr{A}_{f}$ на наборах матриц $A^{00}, A^{01}$ и $A^{1}$ очевидно получаем, что $q_{2}=8, q_{1}=6, q_{3}=27$, таким образом, алгоритм $F_{0}$ правильно определяет ТОФ $f$.

Для произвольной темы $x_{i_{1}} \ldots x_{i_{s}} \bar{x}_{i_{s+1}} \ldots \bar{x}_{i_{t_{j}}}$ определим ее отрицательную часть $\bar{x}_{i_{s+1}} \ldots \bar{x}_{i_{t_{j}}}$ и положительную часть $x_{i_{1}} \ldots x_{i_{s}}$.

Назовем тему допустимой, если она не противоречит текущей фиксации тематических переменных. Более формально: пусть $\Psi\left(x_{1}\right)=y_{q_{1}}, \ldots, \Psi\left(x_{k}\right)=y_{q_{k}}$, то есть переменные $y_{q_{1}}, \ldots, y_{q_{k}}$ являются тематическими. Рассмотрим номера $q_{i_{1}}, \ldots, q_{i_{s}}, i_{s} \in\{1, \ldots, k\}$, такие, что $\left\{q_{i_{1}}, \ldots, q_{i_{s}}\right\}$ является подмножеством множества $m_{2}$ номеров всех помеченных на данном этапе столбцов. Это значит, что каждая из переменных $y_{q_{i_{1}}}, \ldots, y_{q_{i_{s}}}$ фиксирована на этом этапе либо нулем, либо единицей, пусть $y_{q_{i_{1}}}=\sigma_{1}, \ldots, y_{q_{i_{s}}}=\sigma_{s}, \sigma_{j} \in\{0,1\}$, $j \in\{1, \ldots, s\}$. Тогда допустимыми назовем те и только те темы текущего тематического разбиения, в конъюнкции которых не входят $x_{i_{1}}^{\sigma_{1} \oplus 1}, \ldots, x_{i_{s}}^{\sigma_{s} \oplus 1}$. В частности, в рассмотренном выше примере на этапе $A^{1}$ темы $x_{2} x_{3}$ и $\bar{x}_{3}$ являются допустимыми, тема $\bar{x}_{2} x_{3}$ не является допустимой, так как переменная $y_{8}=\Psi\left(x_{2}\right)$ фиксирована единицей. Естественно, алгоритм $F_{0}$ не знает, какие темы текущего тематического разбиения являются допустимыми (так как не знает, какие переменные являются тематическими), но при этом никакой набор, сгенерированный алгоритмом, не может лежать в теме, не являющейся допустимой.

Если тематическое разбиение $R \in \mathscr{R}_{k^{\prime}}$ зависит от переменной $x_{j}, j \in\left\{1, \ldots, k^{\prime}\right\}$ (переменная $x_{j}$ входит в соответствующую ДНФ), но при этом столбец текущей матрицы с номером $q_{j}$, где $y_{q_{j}}=\Psi\left(x_{j}\right)$, является помеченным, то переменную $x_{j}$ назовем фиктивной переменной разбиения $R$. В противном случае будем называть переменную $x_{j}$ существенной. В частности, переменная $x_{2}$ разбиения $x_{2} x_{3} \vee \bar{x}_{3} \vee \bar{x}_{2} x_{3}$ из примера выше является фиктивной, так как $y_{8}=\Psi\left(x_{2}\right)$, а столбец номер 8 является помеченным. При 
этом переменная $x_{3}$ является существенной.

Покажем, что алгоритм $F_{0}$ работает корректно. В силу того, что алгоритм $F_{0}$ есть последовательность вызовов процедуры TSEARCH, мы остановимся на проверке корректности работы именно этой функции. Пусть фиксированы $n, R \in \mathscr{R}_{k^{\prime}}, f \in \Phi_{R}^{n}$. Рассмотрим дерево $\mathscr{D}_{n, R, f, A}$.

Лемма 3. Для любой вериины $v^{\sigma_{1}, \ldots, \sigma_{i}}$ дерева $\mathscr{D}_{n, R, f, A}$ матрица $A_{e}^{\sigma_{1}, \ldots, \sigma_{i}}$, полученная из $A^{\sigma_{1}, \ldots, \sigma_{i}}$ вычеркиванием помеченных строк и столбиов, образует правильное множество наборов.

Доказательство. Покажем это индукцией по номеру уровня дерева $\mathscr{D}_{n, R, f, A}$. На первом уровне существует единственная вершина $v$, приписанная ей матрица $A$ по построению образовывает правильное множество наборов. Пусть $v^{\sigma_{1}, \ldots, \sigma_{i}}-$ некоторая вершина $i$-го уровня. Пусть $r$ - номер разделяющей строки матрицы $A^{\sigma_{1}, \ldots, \sigma_{i-1}}$. Значит, в матрице $A^{\sigma_{1}, \ldots, \sigma_{i}}$ строка с номером $r$ является помеченной. Легко видеть, что непомеченные столбцы матрицы $A^{\sigma_{1}, \ldots, \sigma_{i}}$ пересекают $r$-ю строку либо все по нулям, либо все по единицам. Значит, если в матрице $A_{e}^{\sigma_{1}, \ldots, \sigma_{i}}$ есть 2 равных столбца, то столбцы с такими же номерами равны и в матрице $A_{e}^{\sigma_{1}, \ldots, \sigma_{i-1}}$, что противоречит предположению индукции.

Лемма 4. Для любой допустимой темы разбиения $R^{\sigma_{1}, \ldots, \sigma_{i}}$

(а) среди переменных отрищательной части (если таковые имеются) существует по крайней мере одна существенная;

(б) среди переменных положительной части (если таковые имеются) существует по крайней мере одна существенная.

Доказательство. Проведем доказательство от противного. Без ограничения общности считаем, что в разбиении $R^{\sigma_{1}, \ldots, \sigma_{i}}$ имеется допустимая тема, отрицательная часть которой не содержит существенных переменных (хотя бы одну фиктивную переменную она при этом содержит). Предположим также, что ни одно из разбиений $R, R^{\sigma_{1}}, R^{\sigma_{1}, \sigma_{2}}, \ldots$, $R^{\sigma_{1}, \ldots, \sigma_{i-1}}$ таким свойством не обладает. Итак, имеется тема

$$
x_{1} \ldots x_{s} c_{1}^{l} \ldots c_{i_{l}}^{l} d_{1}^{l} \ldots d_{j_{l}}^{l} \overline{c_{1}^{r}} \ldots \overline{c_{i_{r}}^{r} d_{1}^{r}} \ldots \overline{d_{j_{r}}^{r}},
$$

где $x_{1}, \ldots, x_{s}$ - существенные переменные разбиения $R^{\sigma_{1}, \ldots, \sigma_{i}}, c_{1}^{l}, \ldots, c_{i_{l}}^{l}, c_{1}^{r}, \ldots, c_{i_{r}}^{r}-$ переменные, являющиеся существенными переменными разбиения $R^{\sigma_{1}, \ldots, \sigma_{i-1}}$ и фиктивными переменными разбиения $R^{\sigma_{1}, \ldots, \sigma_{i}}, d_{1}^{l}, \ldots, d_{j_{l}}^{l}, d_{1}^{r}, \ldots, d_{j_{r}}^{r}-$ фиктивные переменные разбиения $R^{\sigma_{1}, \ldots, \sigma_{i-1}}$.

Рассмотрим 2 случая.

(a) Пусть $\sigma_{i}=0$. Тогда тема (3) на самом деле имеет вид

$$
x_{1} \ldots x_{s} d_{1}^{l} \ldots d_{j_{l}}^{l} \overline{c_{1}^{r}} \ldots \overline{c_{i_{r}}^{r} d_{1}^{r}} \ldots \overline{d_{j_{r}}^{r}} .
$$

Рассмотрим тему разбиения $R^{\sigma_{1}, \ldots, \sigma_{i-1}}$, из которой получилась данная. Это, очевидHO,

$$
x_{1} \ldots x_{s} d_{1}^{l} \ldots d_{j_{l}}^{l} \overline{y_{1}}, \ldots, \overline{y_{q}} \overline{c_{1}^{r}} \ldots \overline{c_{i_{r}}^{r} d_{1}^{r}} \ldots \overline{d_{j_{r}}^{r}} .
$$

Как известно, эта тема должна содержать в себе отрицание некоторой переменной, входящей в разделяющую тему разбиения $R^{\sigma_{1}, \ldots, \sigma_{i-1}}$, так как эта тема и разделяющая тема - две элементарные конъюнкции одной ДНФ.

Разберем следующие 4 случая. 
(1) Эта переменная - какая-то из $d_{1}^{l}, \ldots, d_{j_{l}}^{l}, d_{1}^{r}, \ldots, d_{j_{r}}^{r}$. Но это невозможно, так как эти переменные были константами и для разделяющей темы (теми же), и значит, не могут входить в 2 рассматриваемые темы по-разному.

(2) Эта переменная - какая-то из $c_{1}^{r}, \ldots, c_{i_{r}}^{r}$. Это тоже невозможно, так как эти переменные только что стали константами. А переменная может стать константой, только если она не входила в разделяющую тему.

(3) Эта переменная - какая-то из $y_{1}, \ldots, y_{q}$. Это невозможно, так как тема (4) получена из (5), и значит, все эти переменные абсолютно так же входят в разделяющую тему, как они входят в (5).

(4) Эта переменная - какая-то из $x_{1}, \ldots, x_{s}$. Пусть она входит в разделяющую тему с отрицанием. Но тогда она не может входить в тему (4), так как была фиксирована нулем.

Получили противоречие.

(б) Пусть $\sigma_{i}=1$. Значит, имеется тема $x_{1} \ldots x_{s} c_{1}^{l} \ldots c_{i_{l}}^{l} d_{1}^{l} \ldots d_{j_{l}}^{l} \overline{d_{1}^{r}} \ldots \overline{d_{j_{r}}^{r}}$, которая получилась из темы $x_{1} \ldots x_{s} x_{s+1} \ldots x_{s^{\prime}} c_{1}^{l} \ldots c_{i_{l}}^{l} d_{1}^{l} \ldots d_{j_{l}}^{l} \frac{1}{d_{1}^{r}} \ldots \overline{d_{j_{r}}^{r}}$. Последняя тема, очевидно, является допустимой темой разбиения $R^{\sigma_{1}, \ldots, \sigma_{i-1}}$, что противоречит предположению.

Рассмотрим произвольную концевую вершину $v^{\sigma_{1}, \ldots, \sigma_{i}}$ дерева $\mathscr{D}_{n, R, f, A}$ (пусть она принадлежит $i$-му уровню). Пусть $\left\{a_{1}, \ldots, a_{d}\right\}$ - непомеченные наборы матрицы $A^{\sigma_{1}, \ldots, \sigma_{i}}$. Здесь существует 3 варианта:

(a) все темы $\mathscr{A}_{f}\left(a_{1}\right), \ldots, \mathscr{A}_{f}\left(a_{d}\right)$ крайние, причем существует номер $i_{1}$ такой, что $\mathscr{A}_{f}\left(a_{i_{1}}\right)$ - отрицательная крайняя тема, и номер $i_{2}$ такой, что $\mathscr{A}_{f}\left(a_{i_{2}}\right)$ - положительная крайняя тема, $i_{1}, i_{2} \in\{1, \ldots, d\}$;

(б) все темы $\mathscr{A}_{f}\left(a_{2}\right), \ldots, \mathscr{A}_{f}\left(a_{d}\right)$ - одна и та же крайняя тема, а тема $\mathscr{A}_{f}\left(a_{1}\right)$ не является крайней;

(в) все темы $\mathscr{A}_{f}\left(a_{1}\right), \ldots, \mathscr{A}_{f}\left(a_{d}\right)$ - одна и та же крайняя тема.

В случае (в) тема $\mathscr{A}_{f}\left(a_{1}\right)$ допустимая, причем $\mathscr{A}_{f}\left(a_{1}\right)=\mathscr{A}_{f}\left(\neg^{m_{2}}\left(a_{1}\right)\right)$. Таким образом, все переменные, входящие в тему $\mathscr{A}_{f}\left(a_{1}\right)$, являются фиктивными. Это противоречит утверждению леммы 4, и значит, случай (в) невозможен.

Лемма 5. Для любой концевой вериины $v^{\sigma_{1}, \ldots, \sigma_{i}}$ дерева $\mathscr{D}_{n, R, f, A}$ сущзествует единственная переменная $x_{j}, j \in\{1, \ldots, k\}$, входящая во все конъюнкции $\mathscr{A}_{f}\left(a_{1}\right), \ldots, \mathcal{A}_{f}\left(a_{d}\right)$, причем эта переменная является существенной переменной разбиения $R^{\sigma_{1}, \ldots, \sigma_{i}}$.

Доказательство. Рассмотрим случай (а). Существование переменной $x_{j}$ следует из того, что крайние темы - две конъюнкции одной ДНФ. Существенность этой переменной следует из того, что ни одна фиктивная переменная разбиения $R^{\sigma_{1}, \ldots, \sigma_{i}}$ не может одновременно входить в обе крайние темы разбиения $R^{\sigma_{1}, \ldots, \sigma_{i}}$, а единственность - из правильности множества $A_{e}^{\sigma_{1}, \ldots, \sigma_{i}}$ (лемма 3).

Рассмотрим теперь случай (б). Строка $a_{1}$ получена, очевидно, заменой всех элементов некоторой строки $a_{1}^{\prime}$, соответствующих непомеченным столбцам матрицы $A^{\sigma_{1}, \ldots, \sigma_{i}}$, их отрицаниями. При этом $\mathscr{A}_{f}\left(a_{1}^{\prime}\right) \equiv \mathscr{A}_{f}\left(a_{2}\right) \equiv \ldots \equiv \mathscr{A}_{f}\left(a_{d}\right)-$ крайняя тема. По лемме 4 существует по крайней мере одна существенная переменная $x_{j}, j \in\{1, \ldots, k\}$, входящая в конъюнкцию $\mathscr{A}_{f}\left(a_{1}^{\prime}\right)$. В силу правильности множества $A_{e}^{\sigma_{1}, \ldots, \sigma_{i}}$ (лемма 3 ) такая переменная единственна. Кроме того, очевидно, переменная $x_{j}$ входит и в конъюнкцию $\mathscr{A}_{f}\left(a_{1}\right)$ (опять же, из свойств ДНФ, представляющих тематические разбиения). 
Следствие 1. В каждой кониевой вершине дерева $\mathscr{D}_{n, R, f, A}$ определяется ровно одна тематическая переменная.

Доказательство. Действительно, в силу леммы 5 в каждой концевой вершине дерева $\mathscr{D}_{n, R, f, A}$ имеется правильное множество $A_{e}^{\sigma_{1}, \ldots, \sigma_{i}}$ наборов, причем существует единственная переменная, входящая во все темы $\mathscr{A}_{f}\left(a_{1}\right), \ldots, \mathscr{A}_{f}\left(a_{d}\right)$. Согласно алгоритму $F_{0}$, именно эта тематическая переменная и определяется в данной вершине.

Лемма 6. Для любых $R \in \mathscr{R}_{k}, n>k, f \in \Phi_{R}^{n}$ справедливо неравенство

$$
\left.\varphi\left(F_{0}, R, n, f\right) \leqslant 2 k\right] \log _{2} n[+k
$$

Доказательство. Пусть алгоритм $F_{0}$ вызывает процедуру TSEARCH $s$ pa3, $k_{i}-$ число тематических переменных, найденных во время $i$-го вызова процедуры TSEARCH, $i \in\{1, \ldots, s\}$. Легко видеть, что $\sum_{i=1}^{s} k_{i}=k$. Пусть $i$-му вызову процедуры TSEARCH соответствует дерево $\mathscr{D}_{n, R, f, A}^{i}$. Согласно следствию 1 у этого бинарного дерева ровно $k_{i}$ концевых вершин. В каждой из этих вершин алгоритм $F_{0}$ запрашивает не более $] \log _{2} n[+1$ значений ТОФ $f$. В каждой неконцевой вершине алгоритм $F_{0}$ запрашивает не более ] $\log _{2} n$ [ значений ТОФ $f$. Учитывая, что в любом бинарном дереве число неконцевых вершин не превышает числа концевых, получаем, что в $i$-й вызов процедуры TSEARCH алгоритм $F_{0}$ запрашивает не более $\left.2 k_{i}\right] \log _{2} n\left[+k_{i}\right.$ значений ТОФ. Тогда всего может понадобиться не более $\left.\sum_{i=1}^{s}\left(2 k_{i}\right] \log _{2} n\left[+k_{i}\right)=2 k\right] \log _{2} n[+k$ значений ТОФ.

Преобразуем алгоритм $F_{0}$ в алгоритм $F_{1}$ так, чтобы было выполнено неравенство

$$
\left.\varphi\left(F_{1}, R, n, f\right) \leqslant k\right] \log _{2} n[+2 k
$$

Заметим, что алгоритма $F_{0}$ обладает следующим свойством. Рассмотрим вершины $v^{\sigma_{1}, \ldots, \sigma_{i-1}}$ и $v^{\sigma_{1}, \ldots, \sigma_{i-1}, 0}$ некоторого дерева $\mathscr{D}_{n, R, f, A}$, полученного в результате работы алгоритма $F_{0}$ на функции $f \in \Phi_{R}^{n}$. Тогда любая $i$-я строка матрицы $A^{\sigma_{1}, \ldots, \sigma_{i-1}, 0}$ меньше $i$-й строки матрицы $A^{\sigma_{1}, \ldots, \sigma_{i-1}}$, причем если $i$-я строка матрицы $A^{\sigma_{1}, \ldots, \sigma_{i-1}}$ лежит в крайней отрицательной теме разбиения $R^{\sigma_{1}, \ldots, \sigma_{i-1}}$, то $i$-я строка матрицы $A^{\sigma_{1}, \ldots, \sigma_{i-1}, 0}$ лежит в крайней отрицательной теме разбиения $R^{\sigma_{1}, \ldots, \sigma_{i-1}, 0}$. Аналогичная матрица $A^{\sigma_{1}, \ldots, \sigma_{i-1}, 1}$ обладает теми же свойствами с той только разницей, что меньше заменяется на больше. В связи с этим свойством меняем алгоритм $F_{0}$ так, чтобы он не запрашивал значения оператора $\mathscr{A}_{f}$ на наборе, если до этого было запрошено значение оператора на меньшем (большем) наборе, лежащем в положительной (отрицательной) теме. Полученный алгоритм обозначим через $F_{1}$.

Лемма 7. Для любых $n>k$ для тематического разбиения $R \in \mathscr{R}_{k} u$ ТОФ $f \in \Phi_{R}^{n}$ справедливо неравенство

$$
\left.\varphi\left(F_{1}, R, n, f\right) \leqslant k\right] \log _{2} n[+2 k
$$

Доказательство. Рассмотрим некоторое дерево $\mathscr{D}_{n, R, f, A}$ с $k^{\prime} \leqslant k$ концевыми вершинами, полученное в результате работы алгоритма $F_{1}$ на функции $f \in \Phi_{R}^{n}$. Найдем число $\varphi^{\prime}$ значений оператора $\mathscr{A}_{f}$, запрошенных алгоритмом $F_{1}$ в вершинах дерева $\mathscr{D}_{n, R, f, A}$. Рассмотрим в этом дереве два класса цепочек ребер, отрицательный $L$ и положительный $R$ (соответственно сплошные линии на рис. 3 и пунктирные). Пусть $\mu(v)-$ число значений 


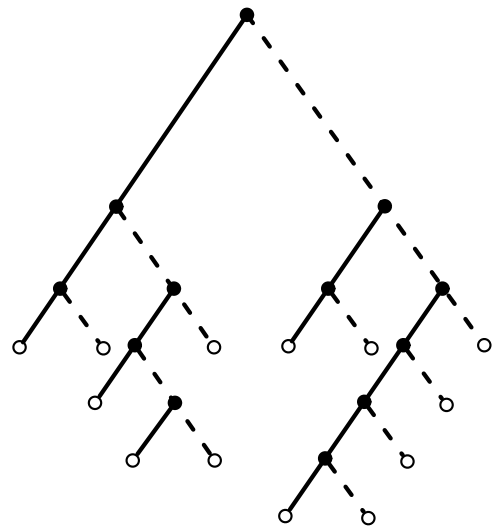

Рис. 3. Возможное решающее дерево

оператора $\mathscr{A}$, запрошенных в вершине $v$ дерева $\mathscr{D}_{n, R, f, A}$. Тогда $\varphi^{\prime}=\sum_{v \in V} \mu(v)$, где $V-$ множество вершин рассматриваемого дерева. Далее,

$$
\varphi^{\prime}=\sum_{l \in L}\left(\sum_{v \in l} \mu^{l}(v)+\mu^{r}\left(v_{l}\right)\right)+\sum_{r \in R}\left(\sum_{v \in r} \mu^{r}(v)+\mu^{l}\left(v_{r}\right)\right)+\sum_{v \in V^{\prime}} 1,
$$

где $\mu^{l}(v)$ - число запрошенных алгоритмом $F_{1}$ наборов вершины $v$, лежащих в отрицательной крайней теме соответствующего этой вершине разбиения, $\mu^{r}(v)-$ число запрошенных алгоритмом наборов вершины $v$, лежащих в положительной крайней теме, $V^{\prime}$ - множество неконцевых вершин рассматриваемого дерева (жирные точки на рис. 3), $v_{l}$ - концевая вершина ветви $l, v_{r}-$ концевая вершина ветви $r$. Последнее слагаемое суммы подсчитывает число наборов, лежащих не в крайних темах.

Оценим $\sum_{v \in l} \mu^{l}(v)+\mu^{r}\left(v_{l}\right)$ для некоторой ветви $l \in L$. Фиксируем некоторое $i \in\{1, \ldots,] \log _{2} n[\}$. Рассмотрим первую вершину ветви $l$, значение оператора $\mathscr{A}_{f}$ на $i$-м наборе которой было запрошено алгоритмом и оказалось отрицательной крайней темой. Согласно описанному выше свойству алгоритма $F_{0} i$-е наборы всех последующих вершин ветви $l$ тоже лежат в отрицательных крайних темах соответствующих тематических разбиений, значения оператора $\mathscr{A}_{f}$ алгоритм $F_{1}$ на них не вычисляет (в отличие от $\left.F_{0}\right)$. Отсюда, очевидно,

$$
\left.\sum_{v \in l} \mu^{l}(v)+\mu^{r}\left(v_{l}\right) \leqslant\right] \log _{2} n[+1
$$

Аналогично,

$$
\left.\sum_{v \in r} \mu^{r}(v)+\mu^{l}\left(v_{r}\right) \leqslant\right] \log _{2} n[+1 .
$$

Легко видеть также, что

$$
\sum_{v \in V^{\prime}} 1=\left|V^{\prime}\right| \leqslant k^{\prime}
$$


Итак,

$$
\begin{aligned}
\varphi^{\prime} & \leqslant \sum_{l \in L}(] \log _{2} n[+1)+\sum_{r \in R}(] \log _{2} n[+1)+k^{\prime} \\
& \left.=(] \log _{2} n[+1)(|L|+|R|)+k^{\prime}=k^{\prime}\right] \log _{2} n\left[+2 k^{\prime} .\right.
\end{aligned}
$$

Теперь, вспоминая, что $\sum k^{\prime}=k$, где сумма берется по всем деревьям, возникшим в результате работы алгоритма $F_{1}$ на функции $f$, получаем требуемое.

Следствие 2. Справедливо неравенство

$$
\varphi(k, n) \leqslant k] \log _{2} n[+2 k .
$$

Доказательство теоремы 1. Согласно лемме 1 и следствию 2, справедливы неравенства

$$
\left.(k-] \log _{2} k[)\right] \log _{2}(n-k+1)[\leqslant \varphi(k, n) \leqslant k] \log _{2} n[+2 k,
$$

из чего следует утверждение теоремы.

\section{Список литературы}

1. Baeza-Yates R., Ribeiro-Neto B., Modern information retrieval. Addison-Wesley, Boston, 1999.

2. Deerwester S., Dumais S. T., Furnas G. W., Landauer T. K., Harshman R., Indexing by latent semantic analysis. J. Amer. Soc. Information Sci. (1990) 41, №6, 391-407.

3. Hofmann T., Probabilistic latent semantic indexing. In: Proc. SIGIR'99, Berkeley, 1999, pp. 55-57.

4. Гасанов Э. Э., Кудрявцев В. Б., Теория хранения и поиска информации. Физматлит, Москва, 2002.

5. Papadimitriou C. H., Raghavan P., Tamaki H., Vempala S., Latent semantic indexing: A probabilistic analysis. J. Comput. Syst. Sci. (2000) 61, №2, 217-235.

6. Коробков В. К., О монотонных функциях алгебры логики. Проблемы кибернетики (1965) 13, 5-28.

Статья поступила 10.07.2006. 